\title{
AhR-mediated, non-genomic modulation of ID01 function
}

\author{
Maria Teresa Pallotta ${ }^{1}$, Francesca Fallarino ${ }^{1}$, Davide Matino $^{1}$, Antonio Macchiarulo $^{2}$ and Ciriana Orabona ${ }^{1}$ * \\ 1 Pharmacology Section, Department of Experimental Medicine, University of Perugia, Perugia, Italy \\ ${ }^{2}$ Department of Pharmaceutical Sciences, University of Perugia, Perugia, Italy
}

Edited by:

Shohei Hori, RIKEN, Japan

Reviewed by:

Wayne Hancock, University of

Pennsylvania School of Medicine,

USA

Liang Zhou, Northwestern University,

USA

Paolo Puccetti, University of Perugia, Italy

*Correspondence:

Ciriana Orabona, Pharmacology Section, Department of Experimental

Medicine, University of Perugia, P.le

Gambuli, Perugia 06132, Italy

e-mail: ciriana.orabona@unipg.it
The evolutionary process has conferred a dual - enzymatic and signaling - function on the ancestral metabolic enzyme indoleamine 2,3-dioxygenase 1 (IDO1), which has long been known for converting the essential amino acid tryptophan (TRP) into neuroactive and immunoactive catabolites (kynurenines). In addition to TRP catabolic activity, phosphorylated immunoreceptor tyrosine-based inhibitory motifs, present in the IDO1 protein, act as docking sites for different molecular partners, which activate positive (transcriptional) or negative (post-translational) modulation of IDO1 protein. The ligand-operated transcription factor aryl hydrocarbon receptor (AhR) contributes to Ido 1 transcription, and it can be operated by both exogenous and endogenous ligands, including L-kynurenine itself, the first byproduct of TRP catabolism. Ligand-bound AhR is also a component of a ubiquitin ligase complex responsible for regulatory proteolysis of different target proteins. Because IDO1 half-life is controlled by the ubiquitin-proteasome system, we here discuss the possibility that $\mathrm{AhR}$, in addition to enhancing Ido1 transcription, contributes to IDO1 regulation by a non-genomic mechanism affecting the protein's half-life.

Keywords: IDO1, AhR, ITIM, ubiquitin ligase complex, tryptophan metabolism, L-kynurenine, immunoregulation, dendritic cells

\section{INTRODUCTION}

Fine tuning of immune reactivity is guaranteed by the recruitment of enzymes with disparate and pleiotropic functions. Indoleamine 2,3-dioxygenase 1 (IDO1) was first recognized as an immune regulator in pregnancy (1), and subsequently, in numerous experimental and clinical settings, including autoimmune diseases, chronic inflammation, transplantation, and neoplasia (2). IDO1 catalyzes degradation of the essential amino acid tryptophan (TRP) along a pathway that causes TRP starvation and yields several biologically active catabolites, collectively known as kynurenines. Similar to other metabolic enzymes, IDO1 is endowed with a second ("moonlighting") function, which makes it a signal-transducing molecule owing to the presence of two immunoreceptor tyrosinebased inhibitory motifs (ITIMs) $(3,4)$. When phosphorylated, those motifs act as docking sites for distinct molecular partners, which can either prolong IDO1's half-life - thus sustaining immunoregulatory effects - or shorten its half-life, so favoring inflammatory responses (5).

The aryl hydrocarbon receptor (AhR), a ligand-activated transcription factor that mediates dioxin toxicity (6), is of vital importance in the regulation of immune responses. Unbound AhR is sequestered in the cytosol by the Hsp90/XAP2 chaperon complex (7-10). Ligand binding to AhR induces conformational changes that promote nuclear translocation of the receptor. In association with the AhR nuclear translocator (Arnt), AhR modulates the transcription of target genes through AhR-responsive elements (AHREs) $(7,8,10)$. A wide array of distinct exogenous and endogenous ligands bind AhR, including indole-containing molecules and TRP metabolites such as L-kynurenine ( $\mathrm{L}-\mathrm{kyn})$, the upstream metabolite generated via TRP degradation $(11,12)$. The immunoregulatory effects mediated by AhR have been known to mostly involve its genomic activity, contributing to Ido1 transcription (13). However, AhR is also involved in a non-genomic pathway, being a component of an atypical ubiquitin ligase complex, which regulates the proteasomal degradation of target proteins (14-16).

\section{REGULATORY PROTEOLYSIS OF ID01 ENZYME}

Intracellular proteins are continually "turning over" as they become hydrolyzed to their constituent amino acids and replaced by new synthesis, which absolves to several important homeostatic functions. Cells contain multiple proteolytic systems to carry out the degradation process and complex regulatory mechanisms, to ensure that the continual proteolytic processes are highly selective. In all tissues, the ubiquitin-proteasome system presides over the degradation of the majority of intracellular proteins (17). In dendritic cells (DCs), under inflammatory conditions, IDO1 itself undergoes proteasomal degradation by associating with suppressor of cytokine signaling 3 (SOCS3) through tyrosine phosphorylated ITIMs present in an IDO1 domain distinct from that mediating its enzymatic function (3).

The crystal structure of the enzyme has indeed been solved in its catalytically inactive conformation (18), unveiling the presence of two folding domains, namely, a large domain and a small domain. The former contains the heme-binding site, forming the catalytic cleft of the enzyme, while the latter contains two highly conserved ITIMs, which, once tyrosine phosphorylated, can act as docking sites for the association with different molecule partners (5). Remarkably, the inspection of the crystal structure of the enzyme (pdb code: 2D0T, 2D0U) shows that the phosphorylable ITIM tyrosines are unexposed to the solvent, and thus, poorly compatible with the experimentally observed interaction with SOCS3. 
Multiple conformational states of enzymes have been reported to play a role in molecular recognition, catalysis, and allosteric modulation (19-21). Compliant to this paradigm, conformational fluctuations of the large and small domains of IDO1 may exist and be triggered by ligand binding and/or by post-translational modifications. Hence, the aforementioned tyrosine phosphorylation of IDO1's ITIMs may promote a specific conformational state of IDO1, amenable the interaction with SOCS3.

Suppressor of cytokine signaling 3 represents the first IDO1 partner identified so far, capable of binding the ITIM docking sites in the enzyme. As a member of the SOCS protein family, it acts as a feedback inhibitor, blocking JAK/STAT signaling in response to pro-inflammatory cytokines, such as IL-6. SOCS3 possesses a Src homology 2 ( $\mathrm{SH} 2$ ) domain, binding phosphotyrosine-containing peptides, and a SOCS box, which recruits an E3 ubiquitin ligase complex and targets several signaling proteins, disparate in nature, for ubiquitination and proteasomal degradation $(22,23)$. We have previously provided evidence that the $\mathrm{SH} 2$ domain in SOCS3, by anchoring phosphorylated IDO1's ITIMs, brings the enzyme close to the E3 ubiquitin complex that ubiquitinates and targets IDO1 for proteasomal degradation. This mechanism provides an explanation for the observed, inverse relationship between SOCS3 and IDO1 expression in DCs. In fact, in DCs lacking SOCS3, the immunoadjuvant effect of the immunogenic fusion protein CD28-Ig is lost, and the latter behaves much like cytotoxic T lymphocyte-associated antigen 4 (CTLA4)-Ig, which is immune suppressive in nature (24). This has been traced to CD28Ig's unique ability to trigger IL-6 and SOCS3 activities, a property unshared by CTLA4-Ig. IL-6-induced SOCS3 is indeed responsible for degrading the protein product of Ido1, whose transcriptional activation is mediated by IFN- $\gamma$ (25). Mutations of the phosphorylable tyrosines in IDO1's ITIM domains completely abolish the ability of the enzyme to bind SOCS3, thus preventing its targeting for proteasomal degradation $(3,26)$.

Therefore, the IDO1/SOCS3 association in DCs represents a molecular mechanism whereby IDO1-positive (IDO1 ${ }^{+}$) DCs, expressing a tolerogenic phenotype, can turn into immunostimulatory antigen presenting cells (APCs), according to environmental needs (3). According to the variety of pathophysiologic contexts that DCs must face, proteasomal degradation of IDO1 could represent a non-genomic mechanism of modulation of the enzyme in order to promptly turn $\mathrm{IDO}^{+}$DCs into immunogenic IDOnegative DCs under conditions in the local microenvironment that require activation of the immune response. Regulatory proteolysis of IDO1 by the ubiquitin-proteasome system may be a more common event than previously appreciated, and it may involve other pathway besides that of IL-6-induced SOCS3 activation.

\section{LIGAND-DEPENDENT E3 UBIQUITIN LIGASE ACTIVITY OF AhR}

Although AhR has traditionally been defined as a transcription factor involved in adaptive xenobiotic and in environmental pollutant responses - including polycyclic and halogenated aromatic hydrocarbons, such as 2,3,7,8-tetrachorodibenzo- $p$-dioxin (TCDD, “dioxin") - the direct transcriptional activity of AhR alone does not fully explain its toxicological and physiological effects. Accumulating evidence suggests that AhR exhibits its regulatory functions by "cross-talking" with a variety of signaling pathways, including estrogen (ER) and androgen (AR) receptors $(27,28)$. In addition to its genomic activity, AhR is also capable of mediating non-genomic effects, by assembling an atypical E3 ubiquitin ligase complex, CUL4B ${ }^{\mathrm{AhR}}$ that includes cullin 4B (CUL4B) $(14,28)$. E3 ubiquitin ligases act in the last step of a sequential reaction, also involving E1 and E2 ligases, and culminating in the ubiquitination of protein substrates.

Similar to the aforementioned ubiquitin ligase activity, mediated by SOCS3 with IDO1 being the target protein, the ubiquitin ligase activity of CUL4B ${ }^{\mathrm{AhR}}$ complex has been reported to target several types of protein for proteolysis. Besides the transcriptional regulation of ERs and ARs, ligand-operating AhR has recently been shown to promote proteasomal degradation of the very same receptors, by assembling the CUL4B ${ }^{\text {AhR }}$ E3 ubiquitin ligase complex (15). Similarly, ligand-based assembly of the CUL4B ${ }^{A h R}$ E3 ligase complex has been found to promote ubiquitination of $\beta$-catenin (16), a transcription factor downstream from the Wnt signaling pathway, leading to proteasomal degradation of $\beta$ catenin in colon tumor cell lines. Interestingly, AhR-deficient mice frequently develop colon tumor with abnormal accumulation of $\beta$-catenin protein. Inversely, administration of AhR ligands efficiently suppressed colon cancer in an established mouse model of familial adenomatous polyposis. These findings suggest that AhR ligands can be used to successfully prevent intestinal tumors, where increased stabilization and accumulation of $\beta$-catenin may be responsible for the initiation of intestinal carcinogenesis. Notably, the substrates of CUL4B ${ }^{\mathrm{AhR}}$ ubiquitin ligase complex - ER, AR, and $\beta$-catenin - all promote cellular proliferation in their target tissues, suggesting that one putative biological role of the ubiquitin ligase function of AhR could be the antiproliferative activity through degradation of those transcription factors, promoting cell proliferation. This raises the possibility of developing selective AhR ligands in cancer therapy, by promoting ubiquitin ligase function.

Besides a direct role of AhR in assembling an ubiquitin ligase complex, there is evidence that AhR may indirectly promote ubiquitination of target proteins. One such recent mechanism has been described for SOCS2-induced proteasomal degradation of tumor necrosis factor receptor-associated factor (TRAF) 6 in a model of Toxoplasma gondii infection. In this study, both L-kyn and the lipoxin $\mathrm{LXA}_{4}$ were found to induce SOCS2-dependent ubiquitination and proteasomal degradation of TRAF6, hindering proinflammatory cytokine expression by DCs. In this case, the mechanism was mediated by the transcriptional activity of AhR, leading to SOCS2 expression that, in turn, promoted TRAF6 polyubiquitination and proteasomal degradation of the adapter proteins (29).

Taken together, the results provide compelling evidence of a prototypic indirect mechanism by which AhR, through AHRE promoter-carrying genes (which include Socs 2 and, interestingly, Socs3) can mediate the proteasomal degradation of target proteins. Notably, L-kyn is a TRP metabolite generated by IDO1 in tolerogenic DCs (30), and it acts as endogenous ligand of AhR in promoting IDO1 phosphorylation, leading to TGF- $\beta$ production (13). Moreover, the interaction of L-kyn with AhR can generate regulatory $\mathrm{T}$ cells (12). Overall, these observations shed light on the crucial relationship between TRP metabolism and both the genomic and non-genomic activities of AhR in modulating immune responses. 


\section{PERSPECTIVE ON AhR-MEDIATED, NON-GENOMIC MODULATION OF ID01}

Several experimental models dissected the tight relationship between TRP metabolism and the activity of AhR in modulating the immune response. It is well established that L-kyn, the upstream metabolite generated by TRP-degrading enzymes, acts as an endogenous AhR ligand, leading to generation of regulatory $\mathrm{T}$ cells, and participating in immune homeostasis $(12,31)$. The IDO1-AhR axis has been described in several settings of immune tolerance, including maternal-fetal tolerance (32), immune suppression induced by several human cancers (33), and endotoxin tolerance (13). Therefore, the molecular dissection of the mechanisms that sustain the immunoregulatory IDO1-AhR axis has become a compelling need.

As a transcription factor, AhR promotes IDO1 expression in response to structurally disparate ligands such as L-kyn - in conventional DCs in a model of endotoxin tolerance (13) - and TCDD, during maturation of bone marrow-derived DCs (34), making Idol as an AhR-responsive gene. Moreover, an autocrine signaling loop involving IL-6, STAT3, and AhR was found to sustain the constitutive expression of IDO1 in human cancer cells (33). The genomic modulation of IDO1 by ligand-operating AhR also involves non-canonical mechanisms mediated by kinase activity. TCDD-activated AhR was independently reported to initiate a rapid non-genomic signaling cascade, culminating in the activation of Src and Erk kinases (35-37). Recently, L-kyn-bound AhR was found to promote IDO1 phosphorylation, through Src kinasemediated activity, which activates the signaling function of IDO1, leading to the de novo synthesis of the enzyme via TGF- $\beta$ production $(4,13)$. Interestingly, in a model of murine vulvovaginal candidiasis an increased expression of AhR was observed in the vagina of both naïve and infected IDO1-deficient mice, suggesting a further mechanism of mutual transcriptional regulation between IDO1, the source of L-kyn, and its sensor AhR (38).

Besides its transcriptional activity, the non-genomic modality of action of AhR could represent a further mechanism whereby TRP metabolism and AhR cross their pathways. Analogous to sex hormone receptors, regulated by AhR in both transcriptionally and non-genomically fashions, IDO1 could represent another substrate for the ubiquitin ligase activity of CUL4B ${ }^{\mathrm{AhR}}$.

Proteasome-mediated degradation of IDO1 has been described in DCs under IL-6-driven pro-inflammatory condition (3). The ubiquitination of the enzyme is mediated by SOCS3 protein that signals the enzyme to the proteasome. The mechanism is particularly active in inflammatory DCs, where SOCS3 is highly expressed and the cells are not required to manifest an immunoregulatory phenotype. Regulatory proteolysis of IDO1 via the ubiquitinproteasome system may represent a non-genomic means of switching off the enzyme. Similar to SOCS3, the CUL4B ${ }^{\text {AhR }}$ complex could promote the ubiquitination of IDO1, targeting it for proteasomal degradation. Physiologically, the non-genomic modulation of IDO1 by ligand-bound AhR could be taken as a typical negative feedback loop of enzyme regulation, where the same trigger (liganded AhR) of its transcriptional expression can also act as a quencher of the protein function, by promoting proteasomal degradation. Such a mechanism would contribute to a fine modulation of IDO1-based immunoregulatory response.
The hypothetical view of AhR-driven proteasomal degradation of IDO1 is based on several observations that include the finding that the enzyme is a proteasome substrate (3). Interestingly, both IL- 6 and SOCS3 (the trigger of the ubiquitination of IDO1) were independently reported to be induced by activated $\operatorname{AhR}(33,39)$. In addition, SOCS2 - a member of the SOCS family in which SOCS3 likewise belongs - is induced by L-kyn-activated AhR, and it promotes the ubiquitination of the adapter protein TRAF6 (29). Overall, these findings suggest that activated AhR, through its transcriptional activity, is capable of inducing all of the components (i.e., IL-6, SOCS3, and IDO1) of a putative feedback loop, promoting intracellular conditions ensuring IDO1 ubiquitination and proteasomal degradation. In addition, post-translational modifications of substrates, such as phosphorylation, typically serve for recruiting E3 ubiquitin ligases. In fact, tyrosine phosphorylation of the two ITIM motifs in the small domain of IDO1 is needed for anchoring SOCS3 protein that bridges E3 ubiquitin ligase (3). Interestingly, IDO1 phosphorylation may be promoted by AhR activity in conventional DCs in a model of endotoxin tolerance (13).

In this hypothetical scenario, AhR would play the canonical role of transcription factor, capable of inducing the stimuli (IL6 and SOCS3) responsible for ubiquitination of IDO1 enzyme, and would promote - through a non-canonical pathway - the phosphorylation of IDO1 required for anchoring SOCS3. It is also likely that the ubiquitin ligase activity ascribed to AhR might directly act on IDO1 as a substrate, bypassing the "bridging" function mediated by SOCS3 (Figure 1). If so, the direct molecular association of CUL4B ${ }^{A h R}$ with IDO1 enzyme should be characterized. All of the previously described complexes of CUL4B ${ }^{A h R}$ with both ER/AR and $\beta$-catenin are localized into the nucleus, as involving the association of ligand-bound AhR with the specific nuclear translocator Arnt. A major question relates to the subcellular location where CUL4B ${ }^{\mathrm{AhR}}$ would bind the substrate IDO1. There is no evidence of a nuclear localization of IDO1 enzyme or of a cytosolic localization of CUL $4 \mathrm{~B}^{\mathrm{AhR}}$. Thus, one should postulate that AhR assembles an ubiquitin ligase complex that involves different molecular partners, not necessarily requiring the nuclear translocation. In this regard, the C-terminus of Hsp70-interacting protein (CHIP), a quality-controlling ubiquitin ligase, reportedly promotes degradation of AhR. Since both CHIP and unliganded AhR are mainly located in the cytosol, the degradation of AhR through CHIP is likely to occur in the cytosol (40).

A second question relates to the nature of the AhR ligand that could target IDO1 as a substrate for ubiquitination. In view of a fine cross-talk between the TRP metabolism and AhR activity, L-kyn and the downstream TRP metabolites could be good candidates for playing this role in a negative feedback loop, aimed at controlling IDO1 enzymatic activity. As it holds true of ER/AR signaling, in which AhR appears to modulate the receptors both positively and negatively, although L-kyn has been shown to transcriptionally induce IDO1 expression via AhR, later in an inflammatory context, the same molecules generated by IDO1 enzymatic activity could also promote IDO1 ubiquitination and proteasomal degradation.

Thus, L-kyn and its derivatives along the kynurenine pathway, exploiting the genomic and non-genomic modality of action of 


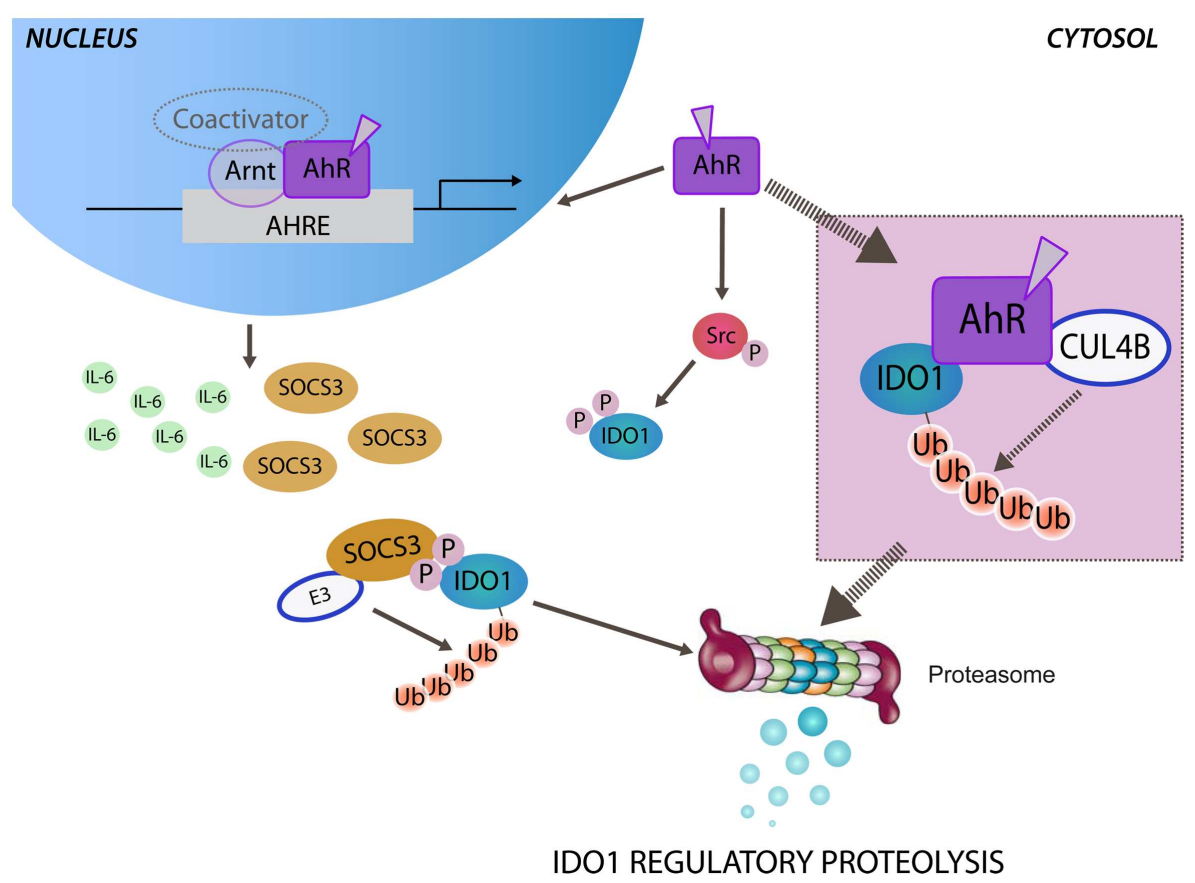

FIGURE 1 | Aryl hydrocarbon receptor-mediated regulatory proteolysis of IDO1. Ligand-operating aryl hydrocarbon receptor (AhR) promotes both genomic and non-genomic activity. After nuclear translocation, ligand-bound AhR dimerizes with the AhR nuclear translocator (Arnt) and activates the transcription of target genes through AhR-responsive elements (AHREs). Both IL-6 and SOCS3 are AhR-responsive genes and may be independently induced by the genomic activity of AhR. In the cytosol SOCS3, by anchoring tyrosine phosphorylated IDO1, brings the enzyme close to the E3 ubiquitin complex (E3) that promotes the polyubiquitination and the proteasomal degradation of IDO1. The non-genomic activity of ligand-bound AhR promotes Src kinase-mediated phosphorylation of IDO1, required for anchoring SOCS3. Ligand-bound AhR can assemble an atypical E3 ubiquitin ligase complex, involving cullin $4 \mathrm{~B}$ (CUL4B), namely, CUL4B AhR . A direct association of CUL4B AhR with IDO1 protein has been prospected (inset) in determining the polyubiquitination and the proteasomal degradation of IDO1. the receptor AhR, could tightly control IDO1 activity in a sort of negative feedback loop. By construing the ubiquitin ligase activity of AhR as a sensor of environmental stress, as suggested in sex hormone signaling (41), several inflammatory adverse effects of dioxin-type ligands of AhR could be, at least in part, attributed to the accelerated degradation of IDO1 that physiologically prevents overreacting responses. In this regard, the appreciation of exogenous/endogenous ligands that selectively activate the nongenomic pathway of AhR might shed light on the biological role of AhR-based modulation of IDO1.

The involvement of the atypical ubiquitin ligase activity of AhR in the quenching of IDO1 activity may represent an attractive therapeutic perspective. Translated into the clinic, the non-genomic control of IDO1 by activated AhR becomes of great interest in neoplasia. The main strategy currently envisioned to tackle IDO1 clinically is by inhibiting its enzymatic activity. The post-translational modification of the enzyme, promoting its ubiquitination and proteasomal degradation, could represent a valid alternative or a complementary approach to the enzymatic inhibition. In this regard, the purine analog, fludarabine, currently used as a chemotherapeutic agent, has recently been proposed to act as a promoter of proteasome-mediated degradation of IDO1 in tumors (42). Noteworthy, the ubiquitin ligase function of AhR has also been contextualized to the antiproliferative activity resulting from proteasomal degradation of transcription factors (ER/AR, $\beta$-catenin) that promote cell proliferation in target tissues. Although IDO1 does not possess a transcriptional activity of its own like other substrates of CUL4B ${ }^{A h R}$, it is noteworthy that, in cancer tissues, IDO1 plays a proliferative action (43-45), and therefore, the putative CUL4 $\mathrm{B}^{\mathrm{AhR}}$-mediated degradation of IDO1 may result in antiproliferative activity.

\section{CONCLUSION}

Dissecting the molecular mechanism of ubiquitin ligase activity of AhR might lead to a better understanding of the diverse biological effects induced by exogenous/endogenous AhR ligands. Specifically, clarifying this mechanism in relationship to the AhR-IDO1 axis might be of great interest in providing innovative IDO1-based therapeutic targets. AhR-mediated non-genomic modulation of IDO1 might provide druggable targets in cancer therapy, in alternative to or in combination with the already available enzyme inhibitors. Thus, the identification of selective "non-toxic" AhR ligands, activating the non-canonical pathway of the receptor, represents an emerging area of research.

\section{ACKNOWLEDGMENTS}

We thank G. Andrielli for digital art and image editing. This work was supported by the Italian Ministry of University and Research (PRIN 2012S47X27 to Ciriana Orabona and Antonio Macchiarulo), Bayer Grants Target Focus Grant no. 2012-03-0630 
(to Francesca Fallarino and Davide Matino), Bayer Early Career Investigator Award (to Davide Matino), and Bayer Special Project Award (to Francesca Fallarino).

\section{REFERENCES}

1. Munn DH, Zhou M, Attwood JT, Bondarev I, Conway SJ, Marshall B, et al. Prevention of allogeneic fetal rejection by tryptophan catabolism. Science (1998) 281:1191-3. doi:10.1126/science.281.5380.1191

2. Puccetti P, Grohmann U. IDO and regulatory T cells: a role for reverse signalling and non-canonical NF-kappaB activation. Nat Rev Immunol (2007) 7:817-23. doi:10.1038/nri2163

3. Orabona C, Pallotta MT, Volpi C, Fallarino F, Vacca C, Bianchi R, et al. SOCS3 drives proteasomal degradation of indoleamine 2,3-dioxygenase (IDO) and antagonizes IDO-dependent tolerogenesis. Proc Natl Acad Sci U S A (2008) 105:20828-33. doi:10.1073/pnas.0810278105

4. Pallotta MT, Orabona C, Volpi C, Vacca C, Belladonna ML, Bianchi R, et al. Indoleamine 2,3-dioxygenase is a signaling protein in long-term tolerance by dendritic cells. Nat Immunol (2011) 12:870-8. doi:10.1038/ni.2077

5. Orabona C, Pallotta MT, Grohmann U. Different partners, opposite outcomes: a new perspective of the immunobiology of indoleamine 2,3-dioxygenase. $\mathrm{Mol}$ Med (2012) 18:834-42. doi:10.2119/molmed.2012.00029

6. Stevens EA, Mezrich JD, Bradfield CA. The aryl hydrocarbon receptor: a perspective on potential roles in the immune system. Immunology (2009) 127:299-311. doi:10.1111/j.1365-2567.2009.03054.x

7. Poellinger L. Mechanistic aspects - the dioxin (aryl hydrocarbon) receptor. Food Addit Contam (2000) 17:261-6. doi:10.1080/026520300283333

8. Hankinson O. The aryl hydrocarbon receptor complex. Annu Rev Pharmacol Toxicol (1995) 35:307-40. doi:10.1146/annurev.pa.35.040195.001515

9. Matsumura F, Vogel CF. Evidence supporting the hypothesis that one of the main functions of the aryl hydrocarbon receptor is mediation of cell stress responses. Biol Chem (2006) 387:1189-94. doi:10.1515/BC.2006.146

10. Mimura J, Fujii-Kuriyama Y. Functional role of AhR in the expression of toxic effects by TCDD. Biochim Biophys Acta (2003) 1619:263-8. doi:10.1016/S03044165(02)00485-3

11. Dinatale BC, Murray IA, Schroeder JC, Flaveny CA, Lahoti TS, Laurenzana EM, et al. Kynurenic acid is a potent endogenous aryl hydrocarbon receptor ligand that synergistically induces interleukin-6 in the presence of inflammatory signaling. Toxicol Sci (2010) 115:89-97. doi:10.1093/toxsci/kfq024

12. Mezrich JD, Fechner JH, Zhang X, Johnson BP, Burlingham WJ, Bradfield CA. An interaction between kynurenine and the aryl hydrocarbon receptor can generate regulatory T cells. J Immunol (2010) 185:3190-8. doi:10.4049/jimmunol. 0903670

13. Bessede A, Gargaro M, Pallotta MT, Matino D, Servillo G, Brunacci C, et al. Aryl hydrocarbon receptor control of a disease tolerance defence pathway. Nature (2014) 511:184-90. doi:10.1038/nature13323

14. Ohtake F, Baba A, Takada I, Okada M, Iwasaki K, Miki H, et al. Dioxin receptor is a ligand-dependent E3 ubiquitin ligase. Nature (2007) 446:562-6. doi:10.1038/nature05683

15. Ohtake F, Fujii-Kuriyama Y, Kato S. AhR acts as an E3 ubiquitin ligase to modulate steroid receptor functions. Biochem Pharmacol (2009) 77:474-84. doi:10.1016/j.bcp.2008.08.034

16. Kawajiri K, Kobayashi Y, Ohtake F, Ikuta T, Matsushima Y, Mimura J, et al. Aryl hydrocarbon receptor suppresses intestinal carcinogenesis in ApcMin/+ mice with natural ligands. Proc Natl Acad Sci U S A (2009) 106:13481-6. doi:10.1073/pnas.0902132106

17. Rock KL, Gramm C, Rothstein L, Clark K, Stein R, Dick L, et al. Inhibitors of the proteasome block the degradation of most cell proteins and the generation of peptides presented on MHC class I molecules. Cell (1994) 78:761-71. doi:10.1016/S0092-8674(94)90462-6

18. Sugimoto H, Oda S, Otsuki T, Hino T, Yoshida T, Shiro Y. Crystal structure of human indoleamine 2,3-dioxygenase: catalytic mechanism of $\mathrm{O} 2$ incorporation by a heme-containing dioxygenase. Proc Natl Acad Sci U S A (2006) 103:2611-6. doi:10.1073/pnas.0508996103

19. Cannon WR, Benkovic SJ. Solvation, reorganization energy, and biological catalysis. J Biol Chem (1998) 273:26257-60. doi:10.1074/jbc.273.41.26257

20. Benkovic SJ, Hammes GG, Hammes-Schiffer S. Free-energy landscape of enzyme catalysis. Biochemistry (2008) 47:3317-21. doi:10.1021/bi800049z
21. Bahar I, Chennubhotla C, Tobi D. Intrinsic dynamics of enzymes in the unbound state and relation to allosteric regulation. Curr Opin Struct Biol (2007) 17:633-40. doi:10.1016/j.sbi.2007.09.011

22. Orr SJ, Morgan NM, Buick RJ, Boyd CR, Elliott J, Burrows JF, et al. SOCS3 targets Siglec 7 for proteasomal degradation and blocks Siglec 7-mediated responses. J Biol Chem (2007) 282:3418-22. doi:10.1074/jbc.C600216200

23. Orr SJ, Morgan NM, Elliott J, Burrows JF, Scott CJ, McVicar DW, et al. CD33 responses are blocked by SOCS3 through accelerated proteasomalmediated turnover. Blood (2007) 109:1061-8. doi:10.1182/blood-2006-05023556

24. Orabona C, Belladonna M, Vacca C, Bianchi R, Fallarino F, Volpi C, et al. Cutting edge: silencing suppressor of cytokine signaling 3 expression in dendritic cells turns CD28-Ig from immune adjuvant to suppressant. J Immunol (2005) 174:6582-6. doi:10.4049/jimmunol.174.11.6582

25. Orabona C, Grohmann U, Belladonna ML, Fallarino F, Vacca C, Bianchi R, et al. CD28 induces immunostimulatory signals in dendritic cells via CD80 and CD86. Nat Immunol (2004) 5:1134-42. doi:10.1038/ni1124

26. Pallotta MT, Orabona C, Volpi C, Grohmann U, Puccetti P, Fallarino F. Proteasomal degradation of indoleamine 2,3-dioxygenase in CD8 dendritic cells is mediated by suppressor of cytokine signaling 3 (SOCS3). Int J Tryptophan Res (2010) 3:91-7. doi:10.4137/IJTR.S3971

27. Tian Y. Ah receptor and NF-kappaB interplay on the stage of epigenome. Biochem Pharmacol (2009) 77:670-80. doi:10.1016/j.bcp.2008.10.023

28. Ohtake F, Baba A, Fujii-Kuriyama Y, Kato S. Intrinsic AhR function underlies cross-talk of dioxins with sex hormone signalings. Biochem Biophys Res Commun (2008) 370:541-6. doi:10.1016/j.bbrc.2008.03.054

29. Mcberry C, Gonzalez RM, Shryock N, Dias A, Aliberti J. SOCS2-induced proteasome-dependent TRAF6 degradation: a common anti-inflammatory pathway for control of innate immune responses. PLoS One (2012) 7:e38384. doi:10.1371/journal.pone.0038384

30. Grohmann U, Fallarino F, Puccetti P. Tolerance, DCs and tryptophan: much ado about IDO. Trends Immunol (2003) 24:242-8. doi:10.1016/S1471-4906(03) 00072-3

31. Nguyen NT, Kimura A, Nakahama T, Chinen I, Masuda K, Nohara K, et al. Aryl hydrocarbon receptor negatively regulates dendritic cell immunogenicity via a kynurenine-dependent mechanism. Proc Natl Acad Sci U S A (2010) 107:19961-6. doi:10.1073/pnas.1014465107

32. Hao K, Zhou Q, Chen W, Jia W, Zheng J, Kang J, et al. Possible role of the 'IDO-AhR axis' in maternal-foetal tolerance. Cell Biol Int (2013) 37:105-8. doi:10.1002/cbin.10023

33. Litzenburger UM, Opitz CA, Sahm F, Rauschenbach KJ, Trump S, Winter $\mathrm{M}$, et al. Constitutive IDO expression in human cancer is sustained by an autocrine signaling loop involving IL-6, STAT3 and the AHR. Oncotarget (2014) 5:1038-51.

34. Vogel CF, Wu D, Goth SR, Baek J, Lollies A, Domhardt R, et al. Aryl hydrocarbon receptor signaling regulates NF-kappaB RelB activation during dendritic-cell differentiation. Immunol Cell Biol (2013) 91:568-75. doi:10.1038/icb.2013.43

35. Dong B, Matsumura F. Roles of cytosolic phospholipase A2 and Src kinase in the early action of 2,3,7,8-tetrachlorodibenzo-p-dioxin through a nongenomic pathway in MCF10A cells. Mol Pharmacol (2008) 74:255-63. doi:10.1124/mol. 107.044669

36. Park S, Mazina O, Kitagawa A, Wong P, Matsumura F. TCDD causes suppression of growth and differentiation of MCF10A, human mammary epithelial cells by interfering with their insulin receptor signaling through $\mathrm{c}$-Src kinase and ERK activation. J Biochem Mol Toxicol (2004) 18:322-31. doi:10.1002/jbt. 20040

37. Mazina O, Park S, Sano H, Wong P, Matsumura F. Studies on the mechanism of rapid activation of protein tyrosine phosphorylation activities, particularly c-Src kinase, by TCDD in MCF10A. J Biochem Mol Toxicol (2004) 18:313-21. doi:10.1002/jbt.20041

38. De Luca A, Carvalho A, Cunha C, Iannitti RG, Pitzurra L, Giovannini G, et al. IL-22 and IDO1 affect immunity and tolerance to murine and human vaginal candidiasis. PLoS Pathog (2013) 9:e1003486. doi:10.1371/journal.ppat. 1003486

39. Brant F, Miranda AS, Esper L, Rodrigues DH, Kangussu LM, Bonaventura D, et al. Immune response profile and development of pathology during Plasmodium berghei Anka infection: the role of the aryl hydrocarbon receptor (AhR). Infect Immun (2014) 82(8):3127-40. doi:10.1128/IAI.01733-14 
40. Morales JL, Perdew GH. Carboxyl terminus of hsc70-interacting protein (CHIP) can remodel mature aryl hydrocarbon receptor (AhR) complexes and mediate ubiquitination of both the AhR and the $90 \mathrm{kDa}$ heat-shock protein (hsp90) in vitro. Biochemistry (2007) 46:610-21. doi:10.1021/bi062165b

41. Ohtake F, Fujii-Kuriyama Y, Kawajiri K, Kato S. Cross-talk of dioxin and estrogen receptor signals through the ubiquitin system. J Steroid Biochem Mol Biol (2011) 127:102-7. doi:10.1016/j.jsbmb.2011.03.007

42. Hanafi LA, Gauchat D, Godin-Ethier J, Possamai D, Duvignaud JB, Leclerc D, et al. Fludarabine downregulates indoleamine 2,3-dioxygenase in tumors via a proteasome-mediated degradation mechanism. PLoS One (2014) 9:e99211. doi:10.1371/journal.pone.0099211

43. Levina V, Su Y, Gorelik E. Immunological and nonimmunological effects of indoleamine 2,3-dioxygenase on breast tumor growth and spontaneous metastasis formation. Clin Dev Immunol (2012) 2012:173029. doi:10.1155/ 2012/173029

44. Mei J, Li MQ, Ding D, Li DJ, Jin LP, Hu WG, et al. Indoleamine 2,3-dioxygenase-1 (IDO1) enhances survival and invasiveness of endometrial stromal cells via the activation of JNK signaling pathway. Int J Clin Exp Pathol (2013) 6:431-44.

45. Moretti S, Menicali E, Voce P, Morelli S, Cantarelli S, Sponziello M, et al. Indoleamine 2,3-dioxygenase 1 (IDO1) is up-regulated in thyroid carcinoma and drives the development of an immunosuppressant tumor microenvironment. J Clin Endocrinol Metab (2014) 99:E832-40. doi:10.1210/jc.2013-3351
Conflict of Interest Statement: The authors declare that the research was conducted in the absence of any commercial or financial relationships that could be construed as a potential conflict of interest. The Review Editor Paolo Puccetti declares that, despite being affiliated to the same institution as authors Maria Teresa Pallotta, Francesca Fallarino, Davide Matino, Antonio Macchiarulo and Ciriana Orabona, the review process was handled objectively.

Received: 18 July 2014; accepted: 24 September 2014; published online: 15 October 2014.

Citation: Pallotta MT, Fallarino F, Matino D, Macchiarulo A and Orabona C (2014) AhR-mediated, non-genomic modulation of IDO1 function. Front. Immunol. 5:497. doi: 10.3389/fimmu.2014.00497

This article was submitted to Immunological Tolerance, a section of the journal Frontiers in Immunology.

Copyright (c) 2014 Pallotta, Fallarino, Matino, Macchiarulo and Orabona. This is an open-access article distributed under the terms of the Creative Commons Attribution License (CC BY). The use, distribution or reproduction in other forums is permitted, provided the original author(s) or licensor are credited and that the original publication in this journal is cited, in accordance with accepted academic practice. No use, distribution or reproduction is permitted which does not comply with these terms. 